\title{
Linx
}

Revue des linguistes de l'université Paris X Nanterre

$7 \mid 1995$

Saussure aujourd'hui

\section{Niveau et distribution d'éléments dans le rapprochement de théories linguistiques}

\section{Rudolf Engler}

\section{OpenEdition}

\section{Journals}

Édition électronique

URL : http://journals.openedition.org/linx/1150

DOI : 10.4000/linx.1150

ISSN : 2118-9692

Éditeur

Presses universitaires de Paris Nanterre

\section{Édition imprimée}

Date de publication : 1 septembre 1995

Pagination : 187-199

ISSN : 0246-8743

\section{Référence électronique}

Rudolf Engler, « Niveau et distribution d'éléments dans le rapprochement de théories linguistiques », Linx [En ligne], 7| 1995, mis en ligne le 13 juillet 2012, consulté le 21 avril 2019. URL : http:// journals.openedition.org/linx/1150 ; DOI : 10.4000/linx.1150

Ce document a été généré automatiquement le 21 avril 2019

Département de Sciences du langage, Université Paris Ouest 


\title{
Niveau et distribution d'éléments dans le rapprochement de théories linguistiques
}

\author{
Rudolf Engler
}

1 S'il y a une théorie linguistique qui aspire à embrasser la totalité des phénomènes du langage, c'est bien celle qu'a développée Ferdinand de Saussure ${ }^{1}$. Je ne dis pas qu'elle les traite, loin de là. Mais dans un système complexe de niveaux et de polarités (interprétés comme des divisions, des oppositions, des antinomies, et ainsi de suite), elle leur fait une place. Ainsi Raffaele Simone a pu dire que la linguistique saussurienne

« ne se propose pas comme une théorie linguistique [parmi d'autres], ni au fond comme une 'théorie' tout court, mais se présente, avec une assurance inconnue jusqu'alors, comme une axiomatique valable pour chaque théorie future voulant s'affirmer comme science, donc comme une 'métathéorie' ; son objectif principal est de fournir une batterie de notions logiquement valables et adéquates aux multiples théories linguistiques qu'elle admet; son but est de spécifier la structure des théories $»^{2}$.

2 Mais bien des lectures du CLG jugent des affirmations saussuriennes comme si elles étaient isolées, limitées en elles-mêmes en méconnaissant le fait qu'ellesforment partie d'un tout, d'un ensemble, d'un 'système serré'. La comparaison de deux articles expliquera la chose, deux articles qui touchent au même sujet - marginal, si l'on veut de l'analyse saussurienne des 'conflits de langue' et qui sont Saussure e $i$ contatti fra lingue ${ }^{3}$ de Vincenzo Orioles, et Bilinguismo e biculturalismo ${ }^{4}$ de Giovanni Freddi.

Orioles - qui se donne la peine de consulter l'édition critique - remarque que Saussure accorde peu de poids à l'emprunt et aux termes artificiels introduits dans la langue, et il cite en particulier CLG/E 405 (N 10) :

«Mais en admettant que chaque [langue connaît des emprunts,] la question serait précisément de savoir : cela est-il un élément régulier sans lequel nous ne saisissons pas le cours naturel des faits linguistiques? Le patois de certaines vallées retirées où l'on constate une introduction de termes artificiels extrêmement peu éloignée de zéro []. Soutiendra-t-on que c'est ce patois qui est hors des conditions régulières du langage et incapable d'en donner une idée; que c'est lui qui demande une étude 
tératologique, comme n'ayant pas subi de mixture? Ces alluvions constituent-elles un élément vital, sans lequel nous ne concevrions pas la perpétuation d'une langue? Non. Donc, ces éléments sont le fait accessoire pour quiconque veut se rendre compte []» Complications de la diversité géographique ( $\$ 2$ al.45) selon laquelle

« les faits dont il a été question dans ce chapitre sont si fréquents qu'ils pourraient passer pour un facteur normal dans l'histoire des langues. Cependant nous ferons ici abstraction de tout ce qui trouble la vue de la diversité géographique naturelle, pour considérer le phénomène primordial, en dehors de toute importation de langue étrangère et de toute formation d'une langue littéraire. Cette simplification schématique semble faire tort à la réalité ; mais le fait naturel doit être d'abord étudié en lui-même » (CLG/E 2923-2925 éd.).

Et il en conclut non seulement que :

«ce qui manque à Saussure est la perception du plurilinguisme comme condition usuelle de la sphère expressive individuelle, comme point d'appui électif et facteur génétique de l'interférence ${ }^{6} "$,

mais il finit par se rallier au jugement de Wandruszka selon lequel Saussure :

« semble ne s'être même pas aperçu de l'évidente incompatibilité entre son schéma anémique d'une langue a priori réduite à un monosystème géométrique et la luxuriance de la polymorphie socioculturelle de nos langues réelles, la profusion des variétés régionales, sociales, culturelles au sein de chacune d'elles, qui exigent, pour une description adéquate, un modèle théorique beaucoup plus riche, beaucoup plus humain que le $\operatorname{sien}^{7} »$.

6 Freddi par contre, dans le but de lier la recherche sur le bilinguisme à une analyse de faits culturels, but qui ne pourrait être compatible avec une vision 'anémique' de la langue, fait entrer en jeu les principes centraux du CLG, considérant que

« une des redécouvertes les plus importantes de la linguistique moderne concerne la nature du signe linguistique qui, comme l'enseigne F. de Saussure, se présente comme une réalité à double face, le signifiant ou image acoustique et le signifié ou concept signifié ${ }^{\prime} »(\mathrm{p} .27)$.

Il prend alors acte du caractère arbitraire du signe linguistique, mais il n'en déduit pas que la langue est une forme vide; il y voit au contraire une possibilité pour la langue de s'imprégner librement des substances culturelles diverses :

«Bien qu'il n'existe aucun lien de nécessité naturelle entre le signifiant et le signifié (c'est pourquoi on parle de l'arbitraire du signe linguistique), pour l'individu qui vient au monde dans une communauté donnée et pour celui qui aborde une seconde langue, le système linguistique qu'il doit s'approprier est tout autre qu'arbitraire. Au contraire, il se présente, fait caractéristique, comme un réseau de rapports prédéterminés rigides : à un système précis de signifiants (les éléments linguistiques) correspond un autre système bien défini de signifiés qui ont leurs racines dans la psychologie et les expériences historico-culturelles du groupe, en un mot dans sa culture » (p.27).

Il tient compte ensuite de la délimitation entre synchronie et diachronie :

«Observée, pour ainsi dire, en filigrane, la langue apparaît comme un précipité de l'histoire du groupe ; affirmation qui ne vaut pas seulement pour le lexique, dans lequel les expériences culturelles de la communauté sont le plus clairement lisibles, mais aussi pour la structuration phonologique et morphosyntaxique. Cette affirmation se vérifie autant du point de vue historique qui illustre son procès évolutif (axe diachronique) que du point de vue fonctionnel qui révèle sa structure à un moment donné (axe synchronique) » (p.28).

Puis il intègre le tout dans la délimitation langue-parole : 
«En tant que 'langue', l'idiome apparaît comme le code de communication, l'ensemble des normes communes à tout le groupe, donc un fait de culture. Au niveau de la 'parole', de l'expression, la culture se manifeste comme coercition qui contient la liberté créatrice de l'individu à l'intérieur des limites imposées par l'efficacité de la communication. Considéré sous ces angles divers, l'idiome s'avère donc être une réalité culturellement déterminée » (p.29).

Enfin, il construit un modèle de bilinguisme composite (deux signifiants pour un signifié) opposé au bilinguisme parallèle (systèmes parallèles de signes constitués par leurs signifiants et signifiés respectifs) qui permet, en dernière analyse, de saisir les interprétations linguistiques concrètes du monde selon chacun des systèmes impliqués :

« Plus haut, on a fait allusion à la nature 'arbitraire' du signe linguistique. (La langue est un 'système de signes' et, à ce titre, elle est l'objet d'une étude sémiologique) Le mot Pferd n'a originairement aucun rapport de nécessité avec l'animal cheval. Cette nature arbitraire en révèle la nature culturelle : le lien entre signifiant et signifié qui, seul, assure la plénitude vitale du signe linguistique, est le résultat d'un pacte sémantique, d'une sorte de contrat social. Contrat qui se renouvelle de moment en moment, allant jusqu'à la répétition du même mot quand le changement des conditions socioculturelles et les exigences d'efficacité de la communication déterminent un 'glissement sémantique', une variation de sens » (p.28).

7 Les deux auteurs arrivent donc à des conclusions diamétralement opposées. Or Orioles aurait dû s'apercevoir par sa citation même - dans des termes comme 'abstraction', 'diversité naturelle', 'phénomène primordial', 'simplification schématique', 'faire tort à la réalité' - qu'une démarche méthodique est en jeu. Il ignore que Saussure déclarait ${ }^{9}$ dans une lettre à Meillet (4 janvier 1894) qu'il n'avait

« pas de plus cher vœu que de n'avoir pas à [s'] occuper de la langue en général », et disait qu'" en dernière analyse seulement le côté pittoresque d'une langue" l'attirait, « celui qui fait qu'elle diffère de toutes les autres comme appar-tenant à un certain peuple ayant certaines origines». ( "C'est ce côté presque ethnographique qui conserve pour moi un intérêt: et précisément, je n'ai plus le plaisir de pouvoir me livrer à cette étude sans arrière-pensée et de jouir du fait particulier tenant à un milieu particulier ».)

Il ne voit donc pas que, dans les passages cités, Saussure, dans une tentative d'organiser la science du langage pour ses étudiants, sépare le plan des phénomènes généraux de celui des faits réels, qu'il établit une hiérarchie entre 'essence', 'nature' des faits ou phénomènes, et la complication accessoire de ces faits dans leur réalisation historique (dont Saussure ne minimise pas l'importance puisque par là-même il l'isole et permet d'en chercher l'explication sur un plan de réalité $\left.{ }^{10}\right)$. Freddi par contre, utilise au maximum les distributions saussuriennes pour développer la théorie dans le sens de ses propres recherches.

La pensée saussurienne, telle qu'elle se dégage des notes et des cours, il faut en convenir, est des plus compliquées. On y reconnaît deux procédés, l'un destructif et l'autre constructif. Le procédé destructif tend vers la 'nature' des choses, et il pourrait probablement être bien intéressant de rassembler tous les textes qui contiennent 'nature' et 'naturel' et d'en faire une analyse. Le procédé constructif vise à une hiérarchisation des phénomènes et leur distribution aux lieux appropriés. Le procédé destructif est perceptible dès 1891 avec le premier texte de linguistique générale que nous avons de Saussure, les leçons d'ouverture à Genève. On ne saurait trop insister sur le fait que dans ces leçons Saussure, sous le couvert d'un rapport sur l'état des choses en linguistique, fait fi de deux notions acquises dans cette science et dans la pensée des gens : l'existence de langues délimitées dans l'espace d'une part et dans le temps de l'autre, tel le français de 
l'hexagone, du XVII ${ }^{\text {ème }}$ au XX ${ }^{\text {ème }}$ siècle ${ }^{11}$. Il n'y a, selon lui, que variation continue dans l'espace et dans le temps: la division en latin et français ne correspond qu'à une dénomination arbitraire et le français n'est en somme que le latin parlé à Paris au XIX ${ }^{\text {ème }}$ siècle; quant à l'espace, si un voyageur allant de Paris à Florence assimilait chacun des changements survenant sur son parcours, il ne quitterait jamais l'état de compréhension 12. La construction prend son essor dans les mêmes textes (voir la définition 'historique' de la langue et la place accordée à l'analogie), mais s'exprime surtout dans les notes $\mathrm{N} 15$, datant de 1897-1900, et dans les trois cours : parce qu'il n'y a pas de langue délimitée temporellement ou spatialement, un concept nouveau de langue doit être élaboré et c'est le concept sémiologique, basé sur la communication : le produit social qui s'établit entre sujets parlants, l'équilibre idiosynchronique toujours labile, dépendant de la masse parlante et du jeu de la parole, des faits internes et externes qui à chaque moment peuvent bouleverser un état donné.

Présenter ainsi l'édifice théorique de Saussure revient à prendre au sérieux les chapitres 'annexes' de la géographie linguistique que personne ne lit plus dans le CLG, mais qui se basent sur trois leçons introductives du cours III $(8,14$ et 17 novembre 1911) et qui reprennent les leçons d'ouverture de 1891. Qui plus est, Saussure, contrairement à son habitude, en a rédigé in extenso de longues parties. Il devait donc y attribuer quelque importance. Cette présentation revient en outre à distinguer dès l'abord deux niveaux, le niveau des faits réels et un niveau de généralisation et d'abstraction. Les faits réels sont complexes ; une analyse s'en trouve proposée sous la forme d'une distribution d'éléments significatifs dans des délimitations théoriques (autres niveaux à considérer au niveau même de l'abstraction). Essayons d'examiner ces délimitations d'une façon peut-être insolite. Il n'y aurait à la base, comme nous venons de le voir, que deux extensions : l'extension du temps, dans lequel tout change, y compris la langue, et l'extension spatiale, sujette elle-même à deux forces opposées, l'esprit de clocher, l'attitude conservatrice, et l' intercourse, l'expansion géographique des traits linguistiques par le 'trafic', le mouvement des hommes et des choses. Dans cette généralisation, cela aussi nous l'avons dit, aucune délimitation de langue n'existerait: il n'y aurait qu'une continuité de traits variés. Au centre des deux extensions, activant les forces, il y a l'homme, les masses parlantes. Ces hommes ont une histoire dans laquelle s'insèrent les faits linguistiques: ce serait le point de vue de la langue dans l'histoire opposée à l'histoire de la langue, les linguistiques externe et interne du CLG. Les faits externes, ce sont toutes ces 'complications' dont il faudra tenir compte une fois résolus les problèmes théoriques. Mais au niveau théorique on ne retiendra pour l'instant qu'une chose : les hommes, par la communication, créent et recréent (à chaque instant), à l'intérieur des deux extensions que nous avons nommées, des produits homogènes de parole qu'on appellera langues. Il n'est pas nécessaire de s'imaginer cette communication dans les conditions du 'clocher', dans une masse homogène usant de paroles identiques. Sinon, à quoi bon l'intercourse? La communication doit en arriver à créer une langue propre au groupe, elle n'a pas besoin de se fonder sur une langue propre au groupe. Et pour cela il faut une force génératrice, la force qui soustend toute communication et qui met les choses en mouvement : la faculté du langage. Cette faculté de langage, souvent confondu dans le terme ambigu de langage tout court, s'active dans la parole et a pour résultat la langue, ce produit de parole qui sera à chaque fois le bien commun d'une masse délimitée de sujets parlants. Dans un certain sens, on pourra dire ici que la masse parlante crée la langue et la langue définit la masse parlante. Cette langue est un système de signes; plus loin en généralisant encore plus 
Saussure dit qu'elle est le signe: l'union d'un signifiant et d'un signifié dans la sanction sociale ${ }^{13}$. On précisera que cet accord est arbitraire, par là même puissant et précaire. Puissant en ce qu'il crée au niveau de la langue une forme virtuelle susceptible d'être chargée au niveau de la parole des références les plus diverses; labile en ce que l'emploi de la parole peut à tout moment influer sur l'accord et l'altérer. Car quelle est la vie du signe dans la parole? Il semble évident que ce n'est pas le signe entier qui est communiqué, mais toujours un signifiant, associé dans la tête de l'émetteur à un signifié précis et qui vient s'associer dans la tête du récepteur au même signifié : mais est-ce le même ? Subissant une dissociation, le signifiant transmis se réfère en même temps à un troisième élément, la référence, la chose dont on parle. Rétablir correctement le signifié veut alors dire pour le récepteur que la chose dont on parle est perçue par lui de la même manière qu'elle l'a été par l'émetteur. Et c'est ce 'percevoir de la même manière' qui est sujet à caution : il y a toujours une marge d'écart, et cette marge d'écart peut mener au changement. La possibilité et la réalisation effective de ce changement évoquent une nouvelle distribution d'éléments : synchronie et diachronie. Comme pour langue et parole, le signe n'est pas le même en synchronie et en diachronie : en synchronie il est l'unité bifaciale, en diachronie il est dissocié. Il y a, comme Saussure le dira dans ses cours, déplacement du lien entre signifiant et signifié, et changement, du signifiant seul, du signifié seul, ou des deux séparément. Or tout changement se produit d'abord, dit Saussure, dans la parole ${ }^{14}$, où nous avions constaté une première dissociation ${ }^{15}$, et se répercute par la sanction sociale sur la langue. Langue et parole, synchronie et diachronie sont donc une distribution croisée d'éléments. Enfin, dans la langue synchronique, il y a la distribution de rapports associatifs et syntagmatiques, où les rapports syntagmatiques reflètent les procédés de la parole linéaire, tandis que les rapports associatifs représentent la classification des rapports, mais une classification qui elle-même ne peut pas ne pas dépendre de la parole, puisqu'elle se fait à tout moment en fonction des mots employés : c'est toujours,le terme employé qui s'accompagne de la présence virtuelle de termes associés. Il y a encore, dans la langue synchronique, la distribution entre éléments motivés et éléments immotivés qu'on distinguera soigneusement de la distribution entre arbitraire et attache naturelle. Tout signe motivé ne l'est que dans le système, par d'autres signes, et en dernière analyse il reste arbitraire.

On est mieux à même de dégager maintenant les qualités et les faiblesses des analyses d'Orioles et Freddi. Orioles identifie la structure profonde de Saussure avec le fait réel, superficiel. Freddi argumente sur la base de la structure profonde et la varie dans le sens du fait réel qu'il veut comprendre. On peut observer les procédés d'Orioles dans beaucoup de critiques faites à Saussure, et il me semble qu'il y a là une règle à observer quand on compare des théories linguistiques: considérer pour l'une et l'autre les niveaux et les distributions en jeu. Ainsi des deux reproches toujours réitérés, selon lesquels Saussure ne considère pas le référent et que sa théorie manque de créativité. Le premier reproche avait été formulé par Ogden et Richards ${ }^{16}$ et se retrouve dès qu'on compare Saussure à Peirce, tandis qu'Ullmann ${ }^{17}$ croit pouvoir intégrer le signe bifacial au modèle triangulaire. Cette tentative est la plus pernicieuse de toutes, parce qu'il est impossible d'ajouter le référent au signe saussurien à un seul et même niveau. Il en résulte que le signe disparaît et qu'on ne raisonne plus qu'en termes de signifié et signifiant : ce que Saussure rejette formellement.

«Item. On ne peut vraiment maîtriser le signe, le suivre comme un ballon dans les airs, avec certitude de le rattraper, que lorsqu'on s'est rendu complètement compte de sa nature double, - nature double, ne consistant nullement dans l'enveloppe et 
pas davantage dans l'esprit, dans l'air hydrogène qu'on y insuffle et <qui> ne vaudrait rien du tout sans l'enveloppe. - Le ballon, c'est le sème, et l'enveloppe le sôme, mais cela est loin de la conception qui dit que l'enveloppe est le signe, et l'hydrogène la signification, sans que le ballon soit rien pour sa part. Il est tout pour l'aérostatier, de même que le sème est tout pour le linguiste » CLG/E 3320.1 les délimitations dans leur ensemble. Si le référent doit apparaître, ce ne peut être qu'au niveau de la parole. Un essai dans ce sens a été fait par Gerold Hilty ${ }^{18}$ :
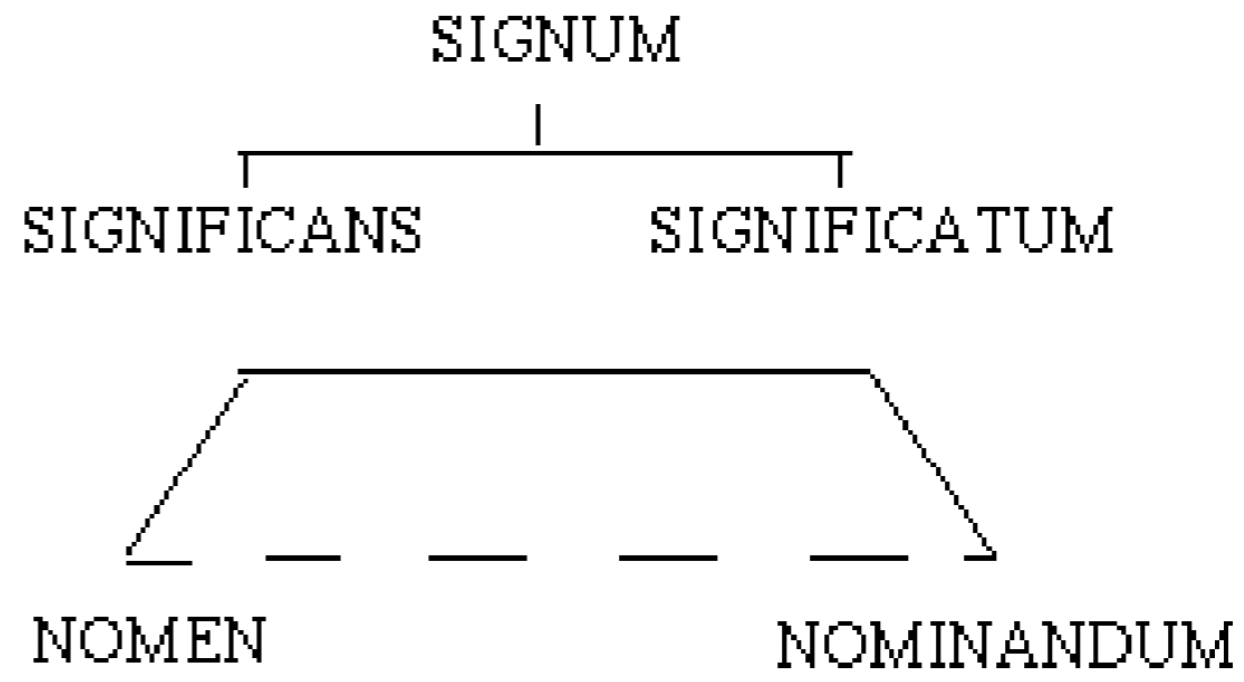

Dans ce modèle SIGNIFICANS et SIGNIFICATUM sont placés sur le plan de la virtualité, NOMEN et NOMINANDUM sur le plan de la réalité, ce qui peut correspondre à langue et parole. On ne touche plus à l'unité du signe. Mais par une correction très simple, une délimitation, celle de la parole, s'ouvre à toutes les recherches touchant aux choses (ainsi, pour une part importante, la sémantique avec son étude des traits distinctifs). A quoi s'ajoute plus généralement un autre avantage: La distinction des niveaux a) garantit la liberté du signe et la possibilité pour la langue de changer de référent, dans chaque acte de parole. Elle empêche que le signe soit fixé sur les qualités innombrables de l'objet actualisé, réel, et préserve sa qualité de valeur virtuelle (ou complexe de valeurs). Elle permet donc au signe et à la langue, à travers la parole et la diachronie, de s'adapter aux situations qui changent. Elle permet aussi b) de maintenir l'unité du signe avec la relation réciproque de signifié et signifiant, empêchant que le signifié devienne l'image du référent. L'emploi métaphorique et métonymique du signe tient à cette particularité. Elle permet enfin c) que l'homme reste psychologiquement libre par rapport au monde. C'est au moins ce que pense François Peraldi dans « Why did Peirce terrorize Benveniste ? "19, qui déclare carrément ${ }^{20}$ que le modèle ternaire de Peirce est utile pour les traitements psychanalytiques, tandis que l'évolution normale de l'homme demande la symbolisation binaire et une négation du référent ${ }^{21}$.

13 Si de son côté la grammaire transformative reproche à la conception saussurienne de la langue d'être taxonomique et de manquer de créativité, on rétorquera que certes Saussure considère la langue comme une classification, mais cette classification touche les procédés syntaxiques (ou syntagmatiques) autant que les unités lexicales (mots, sousunités, syntagmes, phrases toutes faites). Et cette langue 'taxonomique' n'a aucune existence sans ses délimitations complémentaires, le langage et la parole. Or l'exercice de 
la parole (sous l'action de la faculté du langage, à travers la langue) comprend l'actualisation d'un procédé syntagmatique et l'introduction des unités lexicales ${ }^{22}$. Le principe dynamique n'est peut-être pas situé dans la langue (en effet, Saussure la qualifie de passive), mais dans le langage (la faculté de langage) qui fait passer les signes (procédés, unités, sous-unités, syntagmes, phrases toutes faites) de la langue à la parole. C'est la créativité gouvernée par des règles, tout aussi présente chez Saussure que chez Chomsky. Mais le potentiel créatif du modèle saussurien va beaucoup plus loin: il comporte la création de règles; par l'intermédiaire du circuit langue-parole-langue, des éléments de la parole entrent constamment dans la langue et y sont classés et interprétés par la langue : or cette interprétation peut et doit varier selon l'état des matériaux et une interprétation différente peut créer des règles différentes. Les exemples cités par Saussure sont nombreux ; le plus significatif et le plus beau est celui de gant : gantier :

CLG/E 2591 [...] prisonn-ier, gant-ier ; aujourd'hui, il n'y a plus de mot gant, mais gan et la position des termes à associer a changé. Si j'interprète gan, gantier, on ne peut qu'analyser gan-tier ; maintenant, j'ai acquis un genre d'unité qui n'existait pas : tier (si l'on veut, c'est la carte forcée, puisque de par la langue on ne peut <plus> décomposer autrement). De là, de moment en moment je suis exposé <à> appliquer cette unité : clou-tier : est un fait d'analogie, est la création analogique (pas création si l'on veut, puisqu'il a fallu un modèle), est nouveau, ne pouvait pas se former tant que l'on prononçait gant et que l'on coupait gant-ier. »

Mais on peut citer au moins encore meridialis : septentrionalis : septentrional : méridional ${ }^{23}$ et oreos apo eimi : apoeimi oreos ${ }^{24}$. Une fois de plus il faut dépasser la délimitation isolée, considérer la langue dans l'ensemble langage-langue-parole et étudier ensuite les effets du procès indiqué dans la nouvelle délimitation synchronie-diachronie pour comprendre le jeu des structures saussuriennes.

Ces exemples devront suffire ici. Il serait intéressant - mais pas sans difficultés - de poursuivre dans cette voie en comparant d'autres théories pour discerner leurs analogies et leurs différences. Je pense, pour donner des exemples, à quelques très belles pages de Jaberg sur Gilliéron et sa conception de l'étymologie populaire, où nous sommes presque effrayés de retrouver les thèses de l'association mentale saussurienne, ou à la critique peu compréhensible de Bühler - qui pour le reste a si bien saisi l'importance de Saussure dirigée contre le circuit de parole, ou à Croce, Vossler ${ }^{25}$, Spitzer. Comparaisons à faire à condition de se garder d'approuver sans réserve comme aussi de condamner tel auteur, telle théorie, même - j'ironise un peu - Saussure, dont l'édifice est beaucoup plus menacé par ceux qui le limitent à la lettre que par ceux qui essaient de continuer ses lignes de pensée.

\section{NOTES}

1. Kurt Baldinger a placé la conception saussurienne au centre de son article "L'objet de la linguistique: essai d'un modèle linguistique général ", Travaux de Linguistique et de Littérature (Strasbourg) 15, 1977, 379-383.

2. "Introduzione», p. 19s, in: Ferdinand de Saussure, Introduzione al secondo corso di linguistica generale (1908-1909), testo a cura di Robert Godel, edizione italiana a cura di Raffaele Simone, Roma, 
Ubaldini, 1970; nouvelle impression (sous le titre de "La linguistica comme assiomatica in Saussure » in : Il sogno di Saussure, Bari, Laterza, 1992, p. 159-173 [172s]). Je traduis de l'italien.

3. Incontri linguistici 14, 1991, 105-112.

4. Quaderni per la promozione del bilinguismo [QPB] 1, 1973 ; réimpression QPB 23-24, 1979, 25--31.

5. Texte qui n'a pas de correspondance directe dans les sources.

6. Je traduis de l'italien.

7. Italiano d'oggi. Lingua non letteraria e lingue speciali, Trieste 1974, p. 3. Je traduis de l'italien.

8. Je traduis de l'italien.

9. Lettre à Meillet du 4 janvier 1894, cf. Godel, SM, p. 31 et Benveniste, « Lettres de F. de Saussure à Antoine Meillet ", Cahiers Ferdinand de Saussure 21, 1964, 93-125 [95].

10. Si ORIOLES constate ensuite qu'il y a identité de vue entre SAUSSURE et SCHUCHARDT en ce que tous les deux considèrent qu'il n'y a pas lieu de différencier chez le sujet parlant, l'usage de mots empruntés ou autochtones (mais croit devoir opposer Saussure et Schuchardt en ce que l'un se place sur le terrain de la langue, l'autre au point de vue de l'individu), il méconnaît encore une fois les niveaux en jeu : la langue se réduit pour Saussure à l'impression du sujet parlant et pour le fait en question il y a coïncidence (momentanée) entre l'attitude de ce sujet parlant et l'individu de Schuchardt.

11. On a prétendu que l'idée saussurienne d'une synchronie homogène découlait de l'état du français ; or la théorie de SAUSSURE ne vise pas les langues officielles et littéraires qui sont - une fois de plus - une complication de l'état toujours fortuit et changeant d'une langue 'naturelle'.

12. L'image est empruntée à Gaston Paris, mais Saussure lui ôte le caractère anecdotique et il la radicalise.

13. CLG/E 262 SJC La langue [...] n'a d'essentiel que l'union du sens et de l'image acoustique.

14. CLG/E 2522/2560: S’il est vrai qu'on a toujours besoin du trésor de la langue pour parler, réciproquement, tout ce qui entre dans la langue a d'abord été essayé dans la parole un nombre de fois suffisant pour qu'il en résulte une impression durable ; la langue n'est que la consécration de ce qui avait été évoqué par la parole.

15. La dissociation des signifiant et signifié dans la parole n'est pas indiquée expressis verbis par Saussure; je la déduit d'un texte des notes personnelles, selon lequel il y a deux sortes de dissociation, une dissociation dans la diachronie et une seconde à un lieu non précisé. Pour la diachronie elle sera donc affirmée hautement et à différentes reprises.

16. Ogden, C. E; Richards, I. A, The meaning of meaning. A study of influence of language upon thought and of science of symbolism, Londres 1923.

17. Précis de sémantique française, Berne, Francke, 1952. Voir aussi Semantics: an introduction to the science of meaning. Oxford 1952.

18. «Bedeutung als Semstruktur ». Vox Romanica 30/2, 1971, 242-263 [242].

19. Polyphonic linguistics: the many voices of Emile Benveniste, éd. Sylvère Lotringer et Thomas Gora, The Hague, Mouton, 1981 (Semiotica, special supplement), p. 169-179.

20. «In a very short text entitled Negation published in 1925, Freud described the birth of intelligence and the access to language as a process which takes place in two steps, two dissymmetrical moments. - By language, Freud means this system of signs assuming a symbolic function, that is, representing an object necessarily excluded from language itself. - The first moment is an affirmation (Bejahung), which is considered by Freud as an equivalent of the tendency to unify characteristic of Eros. This tendency functions in the attributive judgment as an introjection. - The second moment is a negation (Verneinung), which is not strictly speaking the substitute for or the equivalent of the destructive drives or, as I would prefer to call them, the death drives, but which is a consequence of an expulsion out of the ego due to the death drives. - In the act of judgment, in the intellectual funtion of judging, this expulsion is absolutely necessary to create the distance between the act of thinking, or we should rather say 
language, and the object which ist represented by language for the subject.- In other words, negation is the moment which is absolutely necessary for the appearance of the representative function of language. In still other words, negation is the mythical prerequisite for the appearance of language described by Saussure. [...] - For the psychotic, there is no separation between the real und language, between the object and its symbol, or its signe. There is in fact no reality. In a way, the psychotic floats through a universe without difference, a universe where each sign is sign of another sign infinitely, but where no sign has been anchored to a thing which would not be a sign. - [...] - No Saussurean linguistics can be used to describe such signs, which are not signs of something else bue signs od other signs. And this is probably why Saussurean linguistics always failed when confronted with the language of the psychotic. But if all linguistic systems are at this point useless, Peirce's conception of signs appears like the very conception of the psychotic sign » (p. 172 et 174)

21. Deux autres 'augmentations' malencontreuses du signe saussurien sont signalées dans l'excellent article de Matti Nyman, « Combinability and situations : is the Saussurean sign in need of augmentation? », Suomen kielitieteellisen yhddistyksen vuosikirja (Helsinki) 1989, 32-52.

22. V. Peter Wunderli, «Saussure und die Kreativität », Vox Romanica 33, 1974, 1-32= SaussureStudien 1.3 Kreativität, Tübingen, Narr, 1981, p. 50-74.

23. CLG/E 2592

24. CLG/E 2721s.

25. Cf. Louis Kukenheim, Esquisse historique de la linguistique française et de ses rapports avec la linguistique générale, 2e éd. , Universitaire Pers Leiden, 1966, p. 105, qui, à mon étonnement, rapproche Saussure de Vossler. 